Nota técnica

Volumen 32(1):316-325. Enero-abril, 2021

e-ISSN 2215-3608, doi:10.15517/am.v32i1.40318

http://www.revistas.ucr.ac.cr/index.php/agromeso

\title{
Viabilidad de Ischaemum rugosum Salisb. determinada mediante la prueba de tetrazolio ${ }^{1}$
}

\section{Viability of Ischaemum rugosum Salisb. determined by the tetrazolium test}

\author{
Mary Pamela Portuguez-García², Ana María Rodríguez-Ruiz², Carolina Porras-Martínez, \\ María Isabel González-Lutz ${ }^{4}$
}

1 Recepción: 23 de enero, 2020. Aceptación: 30 de junio, 2020. Este trabajo formó parte del proyecto No B6017 inscrito en la Vicerrectoría de Investigación de la Universidad de Costa Rica, Estación Experimental Agrícola Fabio Baudrit Moreno.

2 Universidad de Costa Rica, Estación Experimental Agrícola Fabio Baudrit Moreno. Programa de Malezas. Alajuela, Costa Rica. mary. portuguez@ucr.ac.cr (autora para la correspondencia; https://orcid.org/0000-0002-3520-7699), amrodriguezster@gmail.com (https://orcid. org/0000-0002-4312-3188).

3 Fundación para el Fomento y Promoción de la Investigación y Transferencia de Tecnología Agropecuaria de Costa Rica, Costa Rica. San José, Costa Rica. cporrasmartinez@gmail.com (https://orcid.org/0000-0003-1157-8011).

4 Universidad de Costa Rica, Escuela de Estadística. San José, Costa Rica. mariaisabel.gonzalezlutz@ucr.ac.cr (https://orcid.org/0000-00023073-7746).

\section{Resumen}

Introducción. La estimación de la viabilidad de Ischaemum rugosum Salisb. con la prueba de tetrazolio es una medida para el estudio de la dinámica biológica de esta maleza. Objetivo. Evaluar los factores que afectan la viabilidad de I. rugosum Salisb. mediante la prueba de tetrazolio. Materiales y métodos. El trabajo se realizó en el Laboratorio Oficial de Análisis de Calidad de Semillas del Centro para Investigaciones en Granos y Semillas de la Universidad de Costa Rica, San José, Costa Rica; en octubre de 2015. Las semillas de I. rugosum Salisb. se sometieron a los siguientes tratamientos previo a la prueba de tetrazolio: almacenamiento de semilla (temperatura ambiente: $23,9^{\circ} \mathrm{C}$ y temperatura refrigerada: $5^{\circ} \mathrm{C}$ ), imbibición (embebida y no embebida), corte de la cariópside (con corte y sin corte) y unidad propagativa (cariópside pequeña, cariópside grande y espiguilla). Se contaron como viables las semillas teñidas de manera uniforme. Resultados. Hubo interacción significativa entre temperatura de almacenamiento y unidad propagativa, la cariópside grande tuvo una mayor ventaja con respecto a cariópside pequeña $(12,18$ a 1$)$ o la espiguilla (181,27 a 1) y este resultado fue más contundente bajo temperatura ambiente. Hubo interacción significativa entre unidad propagativa y corte, todas las semillas requirieron de corte para que el tetrazolio lograra teñir los tejidos, fue más concluyente el efecto en la semilla grande que en la espiguilla (1326,1 a 1) y que en la pequeña (7,39 a 1). La interacción de las temperaturas de almacenamiento e imbibición y corte e imbibición fue significativa. Las semillas sometidas a temperatura ambiente, embebidas (41,26 a 1) y con corte e imbibición $(18,17$ a 1) fueron más viables. Conclusión. La imbibición y el corte facilitaron la tinción, la semilla grande y el almacenamiento en temperatura ambiente influyeron sobre la viabilidad de I. rugosum Salisb.

Palabras clave: malezas, temperatura de almacenamiento, imbibición, corte de semillas. 


\begin{abstract}
Introduction. The estimation of the viability of Ischaemum rugosum Salisb. with the tetrazolium test is a measure for the study of the weed's biological dynamic. Objective. To evaluate the factors that affect the viability of I. rugosum Salisb using the tetrazolium test. Materials and methods. The work was carried out in the Laboratorio Oficial de Analisis de Calidad de Semillas del Centro para Investigaciones en Granos y Semillas of the Universidad de Costa Rica, San José, Costa Rica; in October 2015. I. rugosum Salisb. seeds were subjected to these treatments prior to the tetrazolium test: seed storage (room temperature: $23,9^{\circ} \mathrm{C}$ and refrigerated temperature: $5{ }^{\circ} \mathrm{C}$ ), imbibition (embedded and not embedded), caryopsid cut (with cut and without cut) and propagation unit (small caryopsis, large caryopsis, and spikelet). Seeds stained uniformly were counted as viable. Results. There was a significant interaction between storage temperature and propagation unit, the use of large caryopsis had a greater advantage over the small caryopsis (12.18 to 1 ) or the spikelet (181.27 to 1$)$ and this result was stronger under room temperature. There was a significant interaction between propagation unit and cut, all the seeds required cutting so that tetrazolium could dye the tissues, the effect on the large seed was more conclusive than on the spikelet (1326.1 to 1) and that in the small one ( 7.39 to 1 ). The interaction of storage and imbibition temperatures and cutting and imbibition was significant. The seeds subjected to room temperature, embedded (41.26 to 1) and with cutting and imbibition (18.17 to 1) were more viable. Conclusion. The imbibition and cutting facilitated staining, the large seed and storage at room temperature influenced the viability of I. rugosum Salisb.
\end{abstract}

Keywords: weeds, storage temperature, imbibition, seed cutting.

\title{
Introducción
}

El zacate manchado (Ischaemum rugosum Salisb.) pertenece a la familia Poaceae, es una planta con metabolismo $\mathrm{C}_{4}$ y una maleza anual común en el cultivo del arroz (Giraldo-Cañas, 2010). En Costa Rica se encuentra principalmente en el arroz de secano que se cultiva en el Pacífico Central, especialmente en la zona arrocera de Parrita (Rodríguez, comunicación personal, 2015).

I. rugosum es una maleza competitiva con el cultivo de arroz (Awan et al., 2014). Por lo general, germina después de este, pero logra sobrepasar en tamaño a este cultivo, lo cual genera daños principalmente por competencia y volcamiento. Por otra parte, se han observado fallas en el control de esta maleza con el herbicida bispiribac sodio que es el que normalmente se usa, lo que podría estar relacionado a un posible desarrollo de resistencia a dicho producto (Rodríguez, comunicación personal, 2015). Se ha reportado que I. rugosum Salisb. es resistente a la aplicación de ciertos herbicidas inhibidores de la ACCasa (Heap, 2020).

Dentro del manejo de malezas es importante el conocimiento de la biología de cada especie, de lo cual un aspecto es la germinación (Rao \& Moody 1995; Akanda et al. 1996; Hossain et al., 1999). En muchos casos es muy difícil obtener una germinación uniforme en las malezas, debido a que están influenciadas por la dinámica y la intensidad de la latencia que presentan algunas especies (Benech-Arnold et al., 2000; Vázquez-Yanes \& Cervantes, 1997).

Una forma de realizar análisis de semillas latentes es aplicar la prueba tetrazolio o prueba TZ. El tetrazolio (2,3,5 trifenil tetrazolio cloruro) es un compuesto incoloro que al entrar en contacto con los tejidos vivos que respiran, provoca una reducción de este compuesto en otro que se llama formazán, que es rojo y no se disuelve en los tejidos vivos, por lo tanto, los tiñe, en consecuencia, una coloración roja intensa indica la presencia de células vivas del embrión (Patil \& Dadlani, 2009).

Las condiciones ambientales pueden ser idóneas para que algunas especies mantengan su capacidad para sobrevivir, siempre y cuando estas se almacenen en recipientes herméticos y se proporcionen temperaturas frescas para prolongar su viabilidad (Doria, 2010). En semillas de berenjena (Solanum melongena L.), el almacenamiento 
en condiciones ambientales a $27,0^{\circ} \mathrm{C}$, en un plazo de 150 días de almacenamiento provocó la disminución de la viabilidad en comparación con el almacenamiento a $5{ }^{\circ} \mathrm{C}$. Según algunos autores, este comportamiento se debe a que bajo condiciones ambientales hay mayor temperatura y fluctuación, lo cual no es tolerado por semillas ortodoxas, dado que pierden la calidad fisiológica (Aramendiz-Tatis et al., 2007). En un estudio, almacenar semillas de papaya (Carica papaya L.) a bajas temperaturas $\left(4^{\circ} \mathrm{C}\right.$ ), resultó en una menor germinación (Alonso-Esquivel et al., 2011).

La imbibición es un procedimiento de preacondicionamiento que facilita los cortes y la eliminación de las glumas, lo cual permite la adecuada penetración de la disolución TZ (Rodríguez-Quilón et al., 2008). En las siguientes semillas de la familia Poaceae se aconseja el remojo previo a la aplicación de prueba TZ de la siguiente forma: en arroz y maíz 18 h, en sorgo 16 h y en trigo de 6 a 18 h (Rao et al., 2007).

El zacate manchado se propaga por semilla. La unidad de dispersión de esta especie se denomina espiguilla que en conjunto forma un racimo y conforma la inflorescencia. Cada espiguilla posee un tamaño de 3,0 a 4,5 mm de largo por 1,0 mm de ancho, son de forma triangular en corte transversal, de color amarillo dorado. La primera gluma de la espiguilla sésil es fuertemente corrugada transversalmente. El fruto es una cariópside de 2,5 a 3,0 mm de largo; cada espiguilla puede producir dos nuevas plantas, que corresponden a dos semillas morfológicamente distintas, que en forma natural van en una sola unidad de dispersión (Ocampo-Sánchez, 1985; Pabón, 1983). El eje embrionario da lugar a la radícula (raíz embrionaria), la cual se forma una vez que inician los procesos germinativos (Pérez, 2002). En la parte dorsal de cada cariópside se observa la emergencia de la radícula.

El objetivo de este ensayo fue determinar mediante la prueba de tetrazolio, factores que afectan la viabilidad de I. rugosum Salisb.

\section{Materiales y métodos}

La prueba de tetrazolio en semillas de I. rugosum Salisb. se llevó a cabo en octubre del 2015 en el Laboratorio Oficial de Análisis de Calidad de Semillas del Centro para Investigaciones en Granos y Semillas (CIGRAS) de la Universidad de Costa Rica (UCR), ubicado en la provincia de San José, Costa Rica.

Las semillas utilizadas se cosecharon en noviembre del año 2014 de un lote de arroz que se ubica en Playa Bandera, en el cantón de Parrita de la provincia de Puntarenas, entre las coordenadas 9³0'32,28'” y 842 23'4,82"O. La semilla se colectó en forma aleatoria de todo el lote, de los racimos maduros y se colocó en bolsas plásticas.

Las semillas se trasladaron al Laboratorio de Malezas de la Estación Experimental Agrícola Fabio Baudrit Moreno (EEAFBM), ubicada en la Garita de Alajuela, donde se almacenaron en frascos plásticos herméticos, unas a temperatura ambiente a $23,9{ }^{\circ} \mathrm{C}$ y otras a una temperatura de $5{ }^{\circ} \mathrm{C}$ en una cámara fría.

El arreglo de tratamientos fue factorial, donde los factores fueron: temperatura, unidad propagativa, imbibición y corte; y los niveles fueron: temperatura $\left(5\right.$ y $23,9^{\circ} \mathrm{C}$ ), unidad propagativa (cariópside pequeña, grande y espiguilla) (Figura 1), con y sin imbibición, y con y sin corte. La unidad experimental fue diez semillas. Cada tratamiento se replicó cuatro veces.

Las cariópsides, tanto la grande como la pequeña, se obtuvieron al remover las glumas con una pinza y una aguja de disección, se trabajó con una lupa para evitar daños a las cariópsides.

Para la imbibición de las semillas, estas se envolvieron en una tela tipo organza, que consiste en un tejido originado de la seda, fino y transparente, y se aseguraron con una goma elástica, luego se sumergieron en $20 \mathrm{ml}$ de agua destilada contenidos en un envase plástico de 7,8 cm de largo por 3,6 cm de diámetro, ahí permanecieron 24 $\mathrm{h}$, posteriormente se vació el contenido de cada frasco y se dejaron en ese mismo recipiente en reposo por $24 \mathrm{~h}$.

Tras cumplirse el periodo de reposo se procedió a realizar el corte a las semillas (tanto las que se embebieron como las que no), el corte en la cariópside se hizo en la parte superior de esta y se hizo de forma trasversal, se seccionó 1/3 del endospermo; mientras que en la espiguilla el corte se hizo de forma transversal en 3/5 de la misma 
en la parte inferior que corresponde a una fracción de la base, la cual es una sección que se encuentra endurecida. Para realizar el corte se empleó un bisturí de laboratorio.
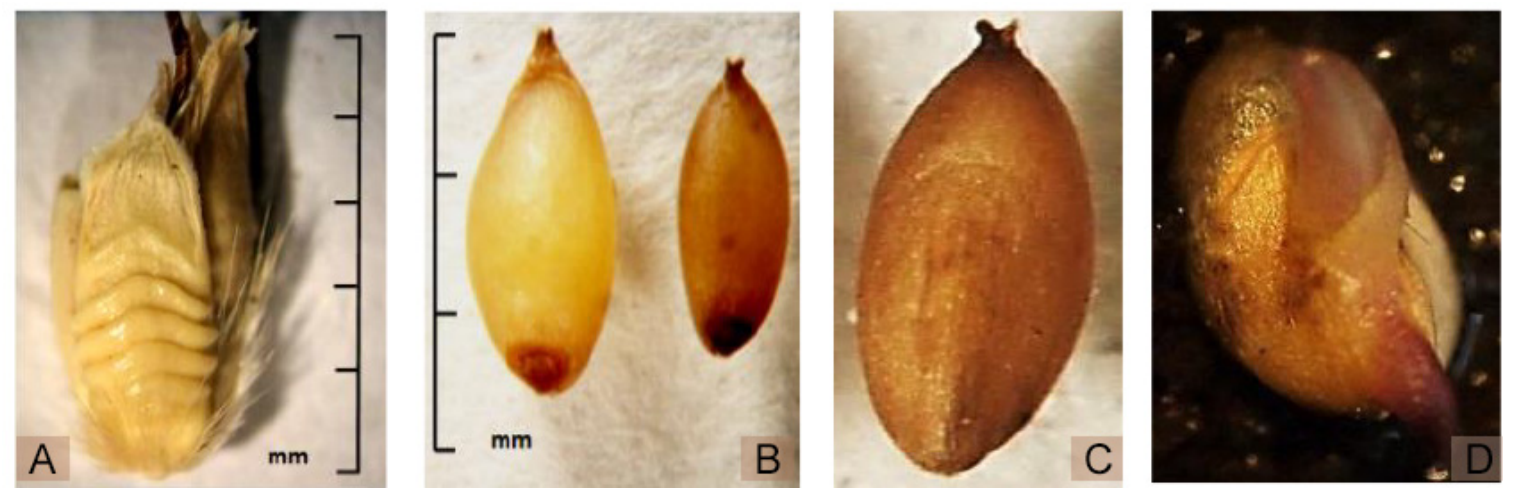

Figura 1. Unidad de dispersión de I. rugsosum Salisb. 40x. A) Espiguilla, B) dos diferentes cariópsides ubicadas en cada unidad de dispersión, una grande y otra pequeña, C) cariópside parte dorsal, D) inicio de emergencia de radícula. Laboratorio Oficial de Análisis de Calidad de Semillas del Centro para Investigaciones en Granos y Semillas (CIGRAS) de la Universidad de Costa Rica. San José, Costa Rica, 2015.

Figure 1. Dispersal unit of I. rugsosum Salisb. 40x A) Spikelet, B) two different caryopsis located in each dispersal unit, one large and the other small, C) carypside dorsal part, D) emergency start of radicle. Laboratorio Oficial de Análisis de Calidad de Semillas del Centro para Investigaciones en Granos y Semillas (CIGRAS) de la Universidad de Costa Rica. San José, Costa Rica, 2015.

La solución de tetrazolio se preparó al $1 \%$ según la Asociación Internacional de Reglas para Pruebas en Semillas (International Rules for Seed Testing Association, 2014). El grupo de semillas correspondiente a cada tratamiento se colocó en un recipiente de vidrio con capacidad de $25 \mathrm{ml}$ y a cada recipiente se le añadió $5 \mathrm{ml}$ de la solución de tetrazolio con una pipeta. Las semillas se mantuvieron en la solución durante $24 \mathrm{~h}$ en una cámara de germinación a $30{ }^{\circ} \mathrm{C}$. Para evitar la exposición del tetrazolio a la luz, los recipientes se introdujeron en una caja plástica que se cubrió por completo con papel aluminio. Posterior a las 24 h de imbibición en TZ, se descartó la solución de tetrazolio contenida en cada recipiente. Para eliminar por completo la solución se efectuaron tres lavados con agua destilada. Posteriormente, las semillas de cada tratamiento se colocaron en platos Petri de $15 \mathrm{~cm}$ de diámetro. Se realizó el conteo de semillas teñidas uniformemente, el cual fue indicativo de que las semillas se encontraban viables. Las semillas de cada tratamiento se revisaron de forma individual en un estereoscopio.

Las semillas que no se examinaron el mismo día en que se realizaron los lavados, se trasladaron a una cámara de almacenamiento a $7{ }^{\circ} \mathrm{C}$ para evitar una posible contaminación y degeneración.

$\mathrm{El}$ análisis de datos se hizo a través de un modelo lineal generalizado (GML) y se seleccionó una regresión logística para determinar los efectos de los cuatro factores utilizando razones de ventaja entre semilla viable y semilla no viable. Se aplicó un modelo con un arreglo factorial completo, pero muchas de las pruebas para las interacciones se indefinieron por falta de variabilidad en la respuesta. Por esta razón, se decidió utilizar un modelo con solamente interacciones dobles. Los análisis se realizaron en el programa estadístico JMP versión 12.

\section{Resultados}

El análisis indicó que hubo interacción doble significativa entre temperatura de almacenamiento y tipo de unidad propagativa $(\mathrm{p}<0,0001)$, tipo de unidad propagativa y corte $(\mathrm{p}<0,0001)$, temperatura de almacenamiento e imbibición $(\mathrm{p}<0,0001)$ y corte e imbibición $(0,0001)$ (Cuadro 1$)$. 
Cuadro 1. Interacciones dobles y su probabilidad asociada de acuerdo con el modelo logístico para la prueba de tetrazolio en semillas de I. rugosum Salisb. Laboratorio Oficial de Análisis de Calidad de Semillas del Centro para Investigaciones en Granos y Semillas (CIGRAS) de la Universidad de Costa Rica. San José, Costa Rica, 2015.

Table 1. Double interactions and their associated probability according to the logistic model for the tetrazolium test in seeds of $I$. rugosum Salisb. Laboratorio Oficial de Análisis de Calidad de Semillas del Centro para Investigaciones en Granos y Semillas (CIGRAS) de la Universidad de Costa Rica. San José, Costa Rica, 2015.

\begin{tabular}{lc}
\hline Interacción & Probabilidad $>$ Chi cuadrado \\
\hline Temperatura de almacenamiento & $<0,0001^{*}$ \\
Tipo de unidad propagativa & $<0,0001^{*}$ \\
Temperatura de almacenamiento * tipo de unidad propagativa & $<0,0001^{*}$ \\
Corte & $<0,0001^{*}$ \\
Temperatura de almacenamiento * corte & $<0,5348$ \\
Tipo de unidad propagativa * corte & $<0,0001^{*}$ \\
Imbibición & $<0,0001^{*}$ \\
Temperatura de almacenamiento * imbibición & $<0,0001^{*}$ \\
Tipo de unidad propagativa *imbibición & $<0,3801$ \\
Corte *imbibición & $<0,0001^{*}$ \\
\hline
\end{tabular}

En la interacción temperatura de almacenamiento y unidad propagativa, las cariópsides grandes mostraron una mayor ventaja de tinción con respecto a las cariópsides pequeñas y la espiguilla, igualmente, las cariópsides pequeñas tuvieron mayor ventaja sobre la espiguilla, esta ventaja fue superior en las semillas que provinieron del almacenamiento a temperatura ambiente en comparación a las de temperatura refrigerada. Cuando se empleó semilla de la condición de temperatura refrigerada, la cariópside grande mostró un $43 \%$ más de tinción que la cariópside pequeña, lo cual sugiere que la semilla de la cariópside grande tuvo mayor viabilidad que la pequeña. En la interacción tipo de unidad propagativa y corte, para el tipo de unidad propagativa ocurrió lo mismo que se describió anteriormente, aunque en este caso al utilizar semilla con corte hubo mayor ventaja de tinción (Cuadro 2) (Figura 2).

Cuadro 2. Razones de ventaja de las interacciones tipo de almacenamiento y tipo de unidad propagativa y tipo de almacenamiento y corte, en semillas de I. rugosum. Laboratorio Oficial de Análisis de Calidad de Semillas del Centro para Investigaciones en Granos y Semillas (CIGRAS) de la Universidad de Costa Rica. San José, Costa Rica, 2015.

Table 2. Advantage reasons of the interactions type of storage and type of propagative unit, and type of storage and cutting, in $I$. rugosum seeds. Laboratorio Oficial de Análisis de Calidad de Semillas del Centro para Investigaciones en Granos y Semillas (CIGRAS) de la Universidad de Costa Rica. San José, Costa Rica, 2015.

\begin{tabular}{llccc}
\hline & \multicolumn{3}{c}{ Razones de ventaja } \\
\cline { 2 - 5 } Tratamiento & & Grande/pequeña & Grande/espiguilla & Pequeña/espiguilla \\
\hline \multirow{2}{*}{ Temperatura de almacenamiento } & Ambiente & $12,18 / 1$ & $181,27 / 1$ & $14,01 / 1$ \\
& Refrigerada & $1,43 / 1$ & $71,52 / 1$ & $49,9 / 1$ \\
\multirow{2}{*}{ Corte } & Sí & $7,39 / 1$ & $1326,1 / 1$ & $179,46 / 1$ \\
& No & $2,36 / 1$ & $10,07 / 1$ & $4,26 / 1$ \\
\hline
\end{tabular}

En el caso de la interacción temperatura de almacenamiento e imbibición, hubo una mayor ventaja para las semillas que se almacenaron a temperatura ambiente y se embebieron, la cual fue de 41,26 contra 1; este efecto de la imbibición también fue mayor con respecto a la semilla refrigerada. Para la interacción corte e imbibición 
también existió una mayor ventaja al someter la semilla a imbibición, y hubo una mayor tinción al emplear semilla con corte (Cuadro 3) (Figura 3).
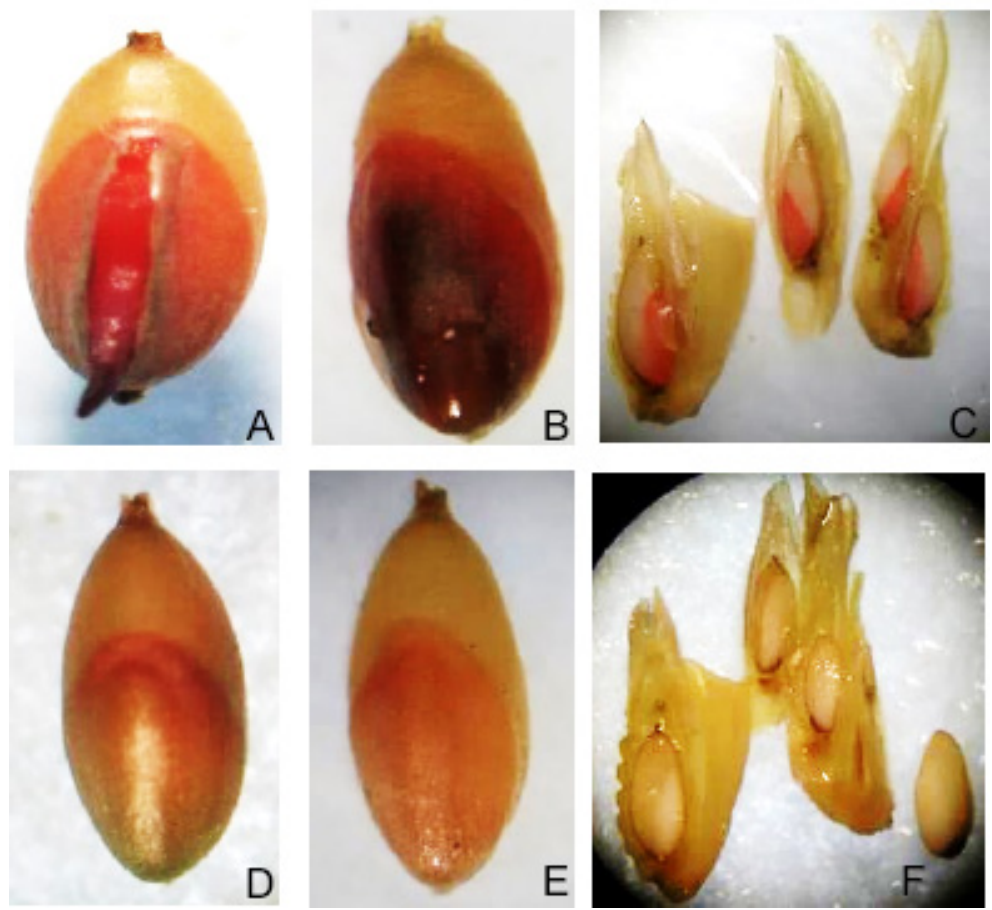

Figura 2. Coloración de las unidades propagativas de I. rugosum Salisb. después de ser sometidas a la prueba de tetrazolio. A) Cariópside grande almacenada a $23,9^{\circ} \mathrm{C}(40 \mathrm{x})$, B) cariópside pequeña almacenada a $\left.23,9{ }^{\circ} \mathrm{C}(40 \mathrm{x}) \mathrm{C}\right)$ espiguilla almacenada a 23,9 ${ }^{\circ} \mathrm{C}(20 \mathrm{x})$, D) cariópside grande almacenada a $5{ }^{\circ} \mathrm{C}(40 \mathrm{x})$, E) cariópside pequeña almacenada a $5{ }^{\circ} \mathrm{C}(40 \mathrm{x})$, F) espiguilla almacenada a $5{ }^{\circ} \mathrm{C}(20 \mathrm{x})$. Laboratorio Oficial de Análisis de Calidad de Semillas del Centro para Investigaciones en Granos y Semillas (CIGRAS) de la Universidad de Costa Rica. San José, Costa Rica, 2015.

Figure 2. Staining of the propagating units of I. rugosum Salisb. after undergoing the tetrazolium test. A) Large caryopside stored at $23.9^{\circ} \mathrm{C}(40 \mathrm{x})$, B) small caryopside stored at $\left.23.9^{\circ} \mathrm{C}(40 \mathrm{x}), \mathrm{C}\right)$ spikelet stored at $23.9^{\circ} \mathrm{C}(20 \mathrm{x})$, D) large caryopsis stored at $5{ }^{\circ} \mathrm{C}(40$ $\mathrm{x})$, E) small caryopsis stored at $5{ }^{\circ} \mathrm{C}$ caryopsis $\left.(40 \mathrm{x}), \mathrm{F}\right)$ spikelet stored at $5{ }^{\circ} \mathrm{C}(20 \mathrm{x})$. Laboratorio Oficial de Análisis de Calidad de Semillas del Centro para Investigaciones en Granos y Semillas (CIGRAS) de la Universidad de Costa Rica. San José, Costa Rica, 2015.

Cuadro 3. Razones de ventaja de las interacciones tipo de almacenamiento e imbibición, y corte en semillas de I. rugosum Salisb. Laboratorio Oficial de Análisis de Calidad de Semillas del Centro para Investigaciones en Granos y Semillas (CIGRAS) de la Universidad de Costa Rica. San José, Costa Rica, 2015.

Table 3. Advantage reasons of the interactions type of storage and imbibition and cutting in I. rugosum Salisb. seeds. Laboratorio Oficial de Análisis de Calidad de Semillas del Centro para Investigaciones en Granos y Semillas (CIGRAS) de la Universidad de Costa Rica. San José, Costa Rica, 2015.

\begin{tabular}{llc}
\hline & & Razones de ventaja \\
\hline Tratamiento & & Embebida/no embebida \\
\hline \multirow{2}{*}{ Temperatura de almacenamiento } & Ambiente & $41,26 / 1$ \\
& Refrigerada & $3,56 / 1$ \\
\hline \multirow{2}{*}{ Corte } & Sí & $18,17 / 1$ \\
& No & $1,57 / 1$ \\
\hline \hline
\end{tabular}



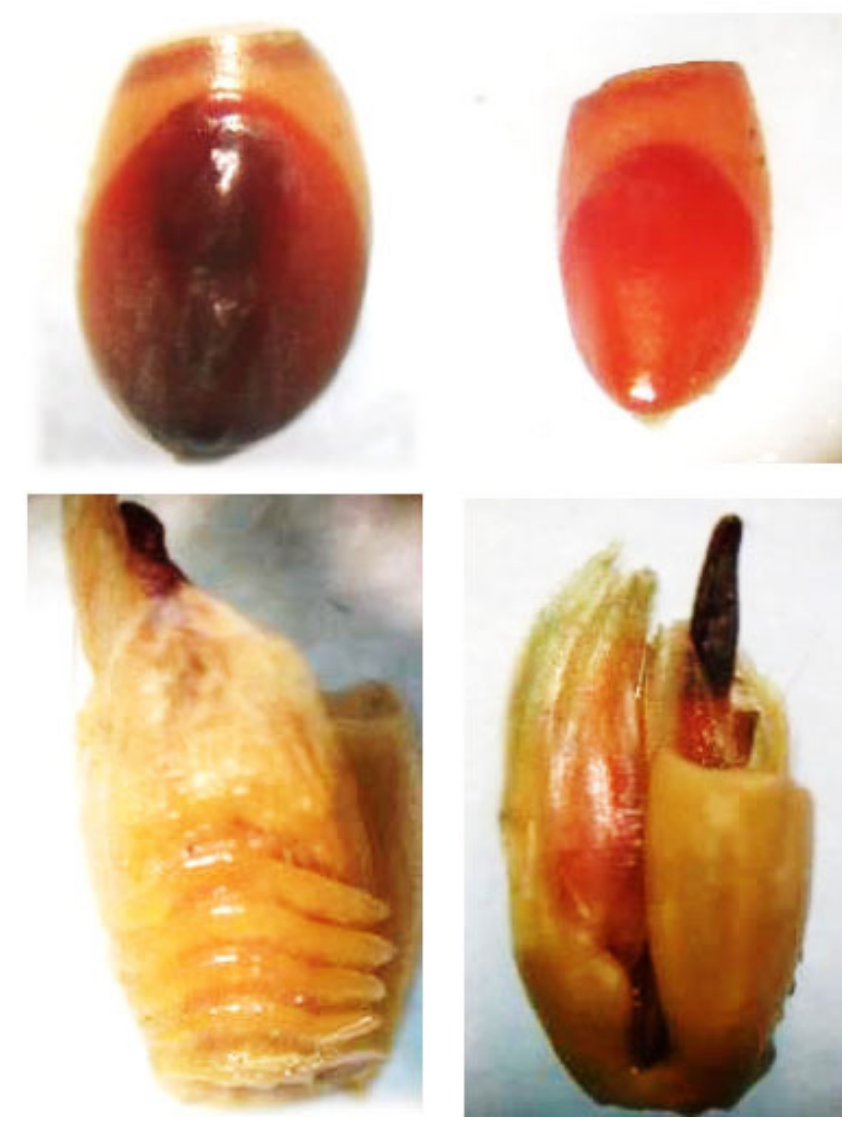

Figura 3. Coloración de las unidades propagativas de I. rugosum Salisb. después de ser sometidas a la prueba de tetrazolio almacenadas a temperatura ambiente, embebidas y con corte. A) Cariópside grande $(40 \mathrm{x})$, B) cariópside pequeña $(40 \mathrm{x})$, C) parte ventral de la espiguilla (30x), D) parte dorsal de la espiguilla (30x). Laboratorio Oficial de Análisis de Calidad de Semillas del Centro para Investigaciones en Granos y Semillas (CIGRAS) de la Universidad de Costa Rica. San José, Costa Rica, 2015.

Figure 3. Staining of the propagating units of I. rugosum Salisb. after being tetrazolium tested and stored at room temperature, embedded, and cut pretreated at room temperature during storage, embedded. A) Large caryopsis (40 x), B) small caryopsis (40 x), C) ventral part of the spikelet (30x), D) dorsal part of the spikelet (30x). Laboratorio Oficial de Análisis de Calidad de Semillas del Centro para Investigaciones en Granos y Semillas (CIGRAS) de la Universidad de Costa Rica. San José, Costa Rica, 2015.

\section{Discusión}

El mayor número de semillas teñidas se obtuvo con la cariópside grande, bajo la condición de temperatura ambiente, ya que dicha tinción fue más notoria y uniforme. Similares resultados reportó (Pabón, 1983), ambas semillas (cariópside grande y pequeña de I. rugosum Salisb.) germinaron, pero la grande lo hizo más rápido y en mayor proporción, lo cual podría tener relación con el mayor número de cariópsides grandes teñidas en comparación con las pequeñas, encontrado en este trabajo.

La mayor viabilidad encontrada en cariópsides grandes de semillas de I. rugosum Salisb. podría vincularse con el tamaño de las semillas, dado que este está relacionado con el vigor, las semillas más grandes poseen mayor ventaja que las pequeñas, ya que la más grande tienen más reservas metabólicas disponibles en los endospermos (Jurado \& Westoby, 1992; Westoby et al., 1992), por ello, el tamaño de las semillas es uno de los factores que determina el éxito competitivo de las especies (Leishman et al., 2000; Westoby et al., 1992). 
Las semillas I. rugosum Salisb almacenadas a $5{ }^{\circ} \mathrm{C}$ no fueron viables. De forma similar ocurrió en semillas de papaya (Carica papaya L.) almacenadas a $4{ }^{\circ} \mathrm{C}$, debido a que bajo tal condición se propicia un alto contenido de agua en las semillas, esto produce su deterioro durante el almacenamiento (Alonso-Esquivel et al., 2011). Contrario a lo que sucede en las semillas de berenjena (Solanum melongera L.) que se favoreció su germinación con una temperatura de almacenamiento a $5{ }^{\circ} \mathrm{C}$, debido a que las fluctuaciones de la temperatura ambiente se asociaron a una pérdida de calidad fisiológica en semillas ortodoxas (Aramendiz-Tatis et al., 2007).

Se obtuvo un efecto más marcado (número de semillas teñidas) al embeber la semilla almacenada a temperatura ambiente, previo a la aplicación de la prueba TZ. Algunos autores han manifestado que las glumas que envuelven la cariópside de I. rugosum Salisb. impiden la germinación (Awan et al., 2014; Jarma-Orozco et al., 2007; Marenco \& Santos, 1999; Pabón, 1983), posiblemente porque las glumas restringen la expansión de embrión debido a su dureza. Por ello, su remoción y el corte pudieron favorecer la tinción, dado que ambos procedimientos expusieron parte del embrión. Al igual que en el presente trabajo, Borza et al. (2007) encontraron que en semillas del género Setaria (Poaceae) se requirió de corte para determinar la viabilidad mediante la prueba TZ.

La tinción de las semillas se propició con el pretratamiento de imbibición, esto también se reportó en especies de familia Poaceae como arroz, maíz, sorgo y trigo (Rao et al., 2007). La imbibición permite el ingreso de la disolución de tetrazolio a los tejidos de las semillas (Rodríguez-Quilón et al., 2008).

\section{Conclusiones}

Mediante la prueba de prueba de tetrazolio, se evidenció el efecto de la temperatura de almacenamiento y del tipo de unidad propagativa sobre la viabilidad de las semillas de I. rugosum. Mientras que, el corte y la imbibición facilitaron la observación de la tinción. La mayor ventaja de tinción siempre se encontró en semillas con la cariópside grande en comparación con la espiguilla y con la cariópside pequeña. La semilla pequeña tuvo mayor ventaja de tinción que utilizar la espiguilla. Por lo que, para obtener una adecuada tinción se deben desprender las glumas de las cariópsides, dado que ambas cariópsides fueron viables, pero la mayor viabilidad se encontró en la cariópside grande.

La imbibición fue necesaria para conocer la viabilidad de las semillas, el efecto fue más influyente en las semillas almacenadas en temperatura ambiente. Bajo esta misma temperatura y en semillas con corte se obtuvo un mayor número de semillas teñidas, lo cual representó las semillas viables.

\section{Referencias}

Akanda, R. U., Mullahey, J. J., \& Shilling, D. G. (1996). Environmental factors affecting germination of tropical soda apple (Solanum viarum). Weed Science, 44(3), 570-574. https://doi.org/10.1017/S0043174500094352

Alonso-Esquivel, M., Ortiz-López, Y., Ramos-Ramírez, R., Oliva-Diaz, H., \& Capote-del Sol, M. (2011). Dormancia en semillas de papaya cv Maradol Roja durante el almacenamiento. Agronomía Mesoamericana, 22(2), 351-357. https:// doi.org/10.15517/am.v22i2.11828

Aramendiz-Tatis, H., Cardona, C., Jarma, A., Robles, J., \& Montalván, R. (2007). Efectos del almacenamiento en la calidad fisiológica de la semilla de berenjena (Solanum melongena L.). Agronomía Colombiana, 25(1), 104-112.

Awan, T. H., Chauhan, B. S., \& Cruz, P. C. S. (2014). Physiological and morphological responses of Ischaemum rugosum Salisb. (Wrinkled Grass) to different nitrogen rates and rice seeding rates. PLoS One, 9(6), e98255. http://doi.org/10.1371/ journal.pone.0098255 
Benech-Arnold, R. L., Sánchez, R. A., Forcella, F., Kruk, B. C., \& Ghersa, C. M. (2000). Environmental control of dormancy in weed seed banks in soil. Field crops research, 67(2), 105-122. https://doi.org/10.1016/S0378-4290(00)00087-3

Berjak, P \& Pammenter, N. W. (2010). Manual de Semillas de Árboles Tropicales [Monografía]. Reforestation, Nurseries, \& Genetics Resources. https://rngr.net/publications/manual-de-semillas-de-arboles-tropicales

Borza, J. K., Westerman, P. R., \& Liebman, M. (2007). Comparing estimates of seed viability in three foxtail (Setaria) species using the imbibed seed crush test with and without additional tetrazolium testing. Weed Technology, 21(2), 518-522. https://doi.org/10.1614/WT-06-110

Doria, J. (2010). Generalidades sobre las semillas: su producción, conservación y almacenamiento. Cultivos Tropicales, 31(1), $74-75$.

Giraldo-Cañas, D. (2010). Distribución e invasión de gramíneas C3 y C4 (Poaceae) en un gradiente altitudinal de los andes de Colombia. Caldasia, 32(1), 65-86.

Heap, I. (2020, July 1). The International Herbicide-Resistant Weed Database. WeedScience. http://www.weedscience.org/ Home.aspx

International Rules for Seed Testing Association. (2014). Seed vigour testing. ISTA.

Hossain, M. A., Ishimine, Y., Akamine, H., Murayama, S., Uddin, S. M., \& Kuniyoshi, K. (1999). Effect of burial depth on emergence of Panicum repens. Weed Science, 47(7), 651-656. https://doi.org/10.1017/S0043174500091281

Jarma-Orozco, A., Arvelaez, J., \& Clavijo, J. (2007). Germinación de Ischaemum rugosum Salisb. en respuesta a estímulos ambientales y químicos. Temas Agrarios, 12(2), 31-41. https://doi.org/10.21897/rta.v12i2.1198

Jurado, E., \& Westoby, M. (1992). Seedling growth in relation to seed size among species of arid Australia. Journal of Ecology, 80(3), 407-416. https://doi.org/10.2307/2260686

Leishman, M. R., Wright, I. J., Moles, A. T., \& Westoby, M. (2000). The evolutionary ecology of seed size. En M. Fenner (Ed.), Seeds: the ecology of regeneration in plant communities (2a ed.) (pp. 31-57). Editorial. CAB International.

Marenco, R. A., \& Santos, R. V. C. D. (1999). Wrinkledgrass and rice intra and interspecific competition. Revista Brasileira de Fisiologia Vegetal, 11(2), 107-111.

Ocampo-Sánchez, R. A. (1985). Incidencia de plantas indeseables en el cultivo de arroz (Oryza sativa) en el cantón de Aguirre y Parrita [Tesis de licenciatura no publicada]. Universidad de Costa Rica

Pabón, R. (1983). Algunos aspectos biológicos de la maleza falsa caminadora (Ischaemum rugosum) en los llanos orientales (Tesis de Maestría, Universidad Nacional de Colombia). Repositorio de la Corporación Colombiana de Investigación Agropecuaria. http://hdl.handle.net/20.500.12324/23911

Patil, V., \& Dadlani, M. (2009). Handbook of seed testing. CABI Publishing.

Pérez, F. 2002. Material vegetal de reproducción: manejo conservación y tratamiento. Junta de Andalucía. http://www. juntadeandalucia.es/medioambiente/consolidado/publicacionesdigitales/80-402_MATERIAL_VEGETAL_DE_ REPRODUCCION__MANEJO_CONSERVACION_Y_TRATAMIENTO/80-402/0_MATERIAL_VEGETAL_DE_ REPRODUCCION.PDF

Rao, A. N., \& Moody, K. (1995, July 24 to 28). Effect of seeding depth and soil moisture regime on emergence of Ischaemum rugosum and Echinochloa glabrescens. ResearchGate. https://www.researchgate.net/publication/286623927_ 
Effect_of_seeding_depth_and_soil_moisture_regime_on_emergence_of_Ischaemum_rugosum_and_Echinochloa_ glabrescens

Rao, N. K., Hanson, J., Dulloo, M. E., \& Ghosh, K. (2007). Manual para el Manejo de Semillas en Bancos de Germoplasma (Manuales para Bancos de Germoplasma No. 8). Bioversity Intenational. https:/www.bioversityinternational.org/ fileadmin/_migrated/uploads/tx_news/Manual_para_el_manejo_de_semillas_en_bancos_de_germoplasma_1261_01.pdf

Rodríguez Quilón, I., Adam, G., \& Durán, J. M. (2008). Ensayos de germinación y análisis de viabilidad y vigor en semillas. Agricultura: Revista Agropecuaria y Ganadera, 78(912), 836-842.

Vázquez-Yanes, C., \& Cervantes, V. (1997). La reproducción de las plantas, semillas y meristemos. Biblioteca Virtual del Instituto Latinoamericano de la Comunicación Educativa. http://bibliotecadigital.ilce.edu.mx/sites/ciencia/volumen3/ ciencia3/157/htm/lcpt157.htm

Westoby, M., Jurado, E., \& Leishman, M. (1992). Comparative evolutionary ecology of seed size. Trends in Ecology \& Evolution, 7(11), 368-372. https://doi.org/10.1016/0169-5347(92)90006-W 